\title{
Feasibility of a Synthetic MR Imaging Sequence for Spine Imaging
}

\author{
(D) M.I. Vargas, (D) M. Drake-Pérez, (DB.M.A Delattre, (D). Boto, (D).-O. Lovblad, and (D) S. Boudabous
}

\begin{abstract}
BACKGROUND AND PURPOSE: Synthetic MR imaging is a method that can produce multiple contrasts from a single sequence, as well as quantitative maps. Our aim was to determine the feasibility of a synthetic MR image for spine imaging.
\end{abstract}

MATERIALS AND METHODS: Thirty-eight patients with clinical indications of infectious, degenerative, and neoplastic disease underwent an MR imaging of the spine (11 cervical, 8 dorsal, and 19 lumbosacral MR imaging studies). The SyntAc sequence, with an acquisition time of 5 minutes 40 seconds, was added to the usual imaging protocol consisting of conventional sagittal T1 TSE, T2 TSE, and STIR TSE.

RESULTS: Synthetic T1-weighted, T2-weighted, and STIR images were of adequate quality, and the acquisition time was $53 \%$ less than with conventional MR imaging. The image quality was rated as "good" for both synthetic and conventional images. Interreader agreement concerning lesion conspicuity was good with a Cohen $\kappa$ of 0.737 . Artifacts consisting of white pixels/spike noise across contrast views, as well as flow artifacts, were more common in the synthetic sequences, particularly in synthetic STIR. There were no statistically significant differences between readers concerning the scores assigned for image quality or lesion conspicuity.

CONCLUSIONS: Our study shows that synthetic MR imaging is feasible in spine imaging and produces, in general, good image quality and diagnostic confidence. Furthermore, the non-negligible time savings and the ability to obtain quantitative measurements as well as to generate several contrasts with a single acquisition should promise a bright future for synthetic MR imaging in clinical routine.

$\mathbf{R}$ adiologists base their diagnoses on morphologic and, increasingly more, quantitative imaging. The current trend in imaging is to reach a diagnosis based on not only morphologic and qualitative evaluation but also methods that can provide quantitative information. Quantitative imaging can be achieved by 2 different techniques: synthetic (used in clinical practice) and fingerprinting (used solely for research purposes). ${ }^{1,2}$ Synthetic MR imaging is a method that can produce multiple contrasts from a single sequence, as well as quantitative T1, T2, STIR, and protondensity maps. This offers the possibility of shortening the study duration and the option of relying on more objective parameters to reach a diagnosis. Recently, this emerging technique has been

Received April 9, 2018; accepted after revision May 29.

From the Division of Diagnostic and Interventional Neuroradiology (M.I.V., J.B. K.-O.L.), Geneva University Hospitals and Faculty of Medicine of Geneva, Geneva, Switzerland; Department of Radiology (M.D.-P.), University Hospital Marqués de Valdecilla, IDIVAL, Santander, Spain; and Division of Radiology (B.M.A.D., S.B.), Geneva University Hospitals, Geneva, Switzerland.

Please address correspondence to Maria Isabel Vargas, MD, Geneva University Hospitals, Division of Diagnostic and Interventional Neuroradiology of Geneva University Hospitals, DISIM, Rue Gabrielle-Perret-Gentil 4, 1211 Genève 14, Switzerland; e-mail: maria.i.vargas@hcuge.ch

http://dx.doi.org/10.3174/ajnr.A5728 applied to several brain diseases in adults ${ }^{3,4}$ and children, ${ }^{5-7}$ but to our knowledge, it has never been used in spine imaging. The purpose of this work was to apply synthetic MR imaging to the spine ${ }^{6}$ and spinal cord and to compare the overall image quality, diagnostic confidence, and lesion conspicuity produced by synthetic MR imaging with conventional sequences.

\section{MATERIALS AND METHODS \\ Patients}

The local ethics committee on research involving humans approved this study (CCER 2016-1821).

A synthetic MR image was added to our usual spine imaging protocol in 38 patients referred to our institution with suspicion of multiple sclerosis or degenerative, infectious, and neoplastic diseases, or for postsurgical follow-up. Exclusion criteria were children, pregnant women, and motion artifacts on the images.

\section{Image Acquisition}

Synthetic imaging was performed in addition to the conventional sequences (T1, FSE, T2, proton-density, and STIR) used in daily clinical practice at our hospital. The patients were 
scanned on an Ingenia 1.5T scanner (Philips Healthcare, Best, the Netherlands).

The SyntAc sequence (Philips Healthcare) is based on a turbo spin-echo acquisition with a saturation pulse of $120^{\circ}$, four TIs, and 2 TEs, producing 8 images with different contrasts. These images are then used by the SyMRI software, Version 8 (SyntheticMR, Linköping, Sweden $)^{8}$ to generate T1, T2, and proton-density quantitative images and to create synthetic T1, T2, and STIR contrasts with specific TEs, TRs, and TIs, which are chosen in postprocessing.

The sequence parameters were the following: sagittal orientation, FOV $=200 \times 321 \mathrm{~mm}$, acquisition (reconstruction), voxel size $=0.89 \times 1.48 \mathrm{~mm}(0.71 \times 0.72 \mathrm{~mm}), 15$ contiguous $4-\mathrm{mm}$ thickness slices, $\mathrm{TE}=11 / 100 \mathrm{~ms}, \mathrm{TR}=2485 \mathrm{~ms}$, TSE factor $=12$, sensitivity encoding acceleration factor $=2$. The acquisition time of the synthetic sequence is 5 minutes 40 seconds. Synthetic images were generated with matching TE/TR parameters and compared with the conventional sequences (T1, T2, and STIR when available) with an imaging acquisition time of 11 minutes 20 seconds. TI was chosen to maximize suppression of the fat signal.

Two sets of images were created for each patient: conventional and synthetic sequences.

\section{Image Evaluation}

Two qualified readers, with 15 and 11 years of experience, respectively, reviewed the images. Image quality was evaluated on conventional T1, T2, and STIR images (when available) and on synthetic images for each patient in random order. Image quality was rated as "poor," "fair," or "good" (scores of 1, 2, and 3, respectively). The presence or absence of lesions was also assessed. Subsequently, both readers rated their degree of confidence regarding the presence or absence of a lesion, which was recorded as "certain" ( score $=1)$ or "not certain" $($ score $=0)$. The presence or absence and the type of artifacts were also evaluated.

\section{Statistical Analysis}

The medians of the scores for image quality and lesion conspicuity obtained with each method (synthetic versus conventional) were compared using the Wilcoxon test. The scores assigned by each reader were compared by the same method. Interreader agreement regarding the certainty of the presence or absence of a lesion was evaluated by the Cohen $\kappa$. All statistics were performed with RStudio (Version 3.3.2; http://rstudio.org/download/desktop).

\section{RESULTS}

A total of 38 patients were included in this study (22 males, 16 females; mean age, 57 years; age range, 17-87 years). Clinical indications for MR imaging were as follows: degenerative pathology, multiple sclerosis, postsurgical follow-up, and diagnosis or follow-up of tumors. Eleven cervical, 8 dorsal, and 19 lumbosacral MR imaging studies were performed.

Image quality was rated as good in synthetic and conventional images by both readers. However, there was a statistically significant difference in the scores assigned for image quality by reader 2 $(P=.02)$ but not reader $1(P=.053)$. Figure 1 shows the distribution of scores for different methods and readers. Although the medians are similar, the synthetic method produced more "fair"

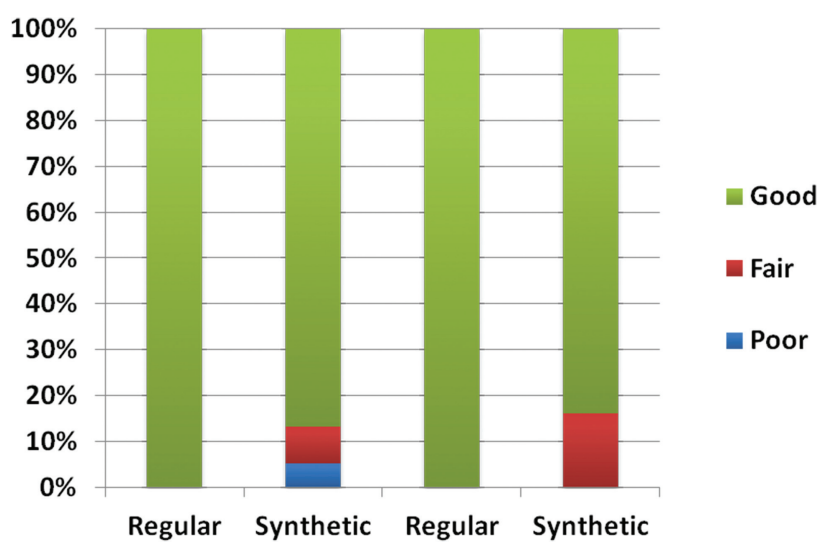

FIG 1. Distribution of scores for image-quality ratings for readers 1 (left) and 2 (right).

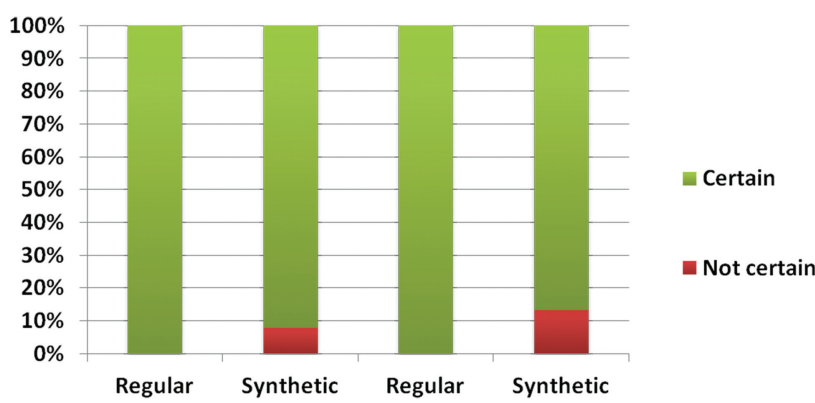

FIG 2. Distribution of scores for certainty of the presence or absence of a lesion for readers 1 (left) and 2 (right).

and "poor" scores ( $n=3$ and $n=2$, respectively, for reader 1 and $n=6$ and $n=0$ for reader 2 ).

There was agreement between readers in identifying the presence or absence of lesions in all cases, except 1 case for reader 1 and 2 cases for reader 2 . In these 3 cases, the lesions were not detected on the synthetic sequence but were easily identified on the conventional sequences. Lesions that were not present on the conventional images were also not incorrectly detected on the synthetic images (no false-positives).

The relative frequencies of certainty for the presence or absence of a lesion, with a median score of 1 , are shown in Fig 2. Interreader agreement was good with a Cohen $\kappa$ of 0.737 , but there were cases in which there was discordance between the synthetic and conventional images ( $n=3$ for reader $1, n=5$ for reader 2). This difference was not statistically significant for reader $1(P=.149)$, but it was significant for reader $2(P=.04)$.

Artifacts consisting of white pixels/spike noise across contrast views and flow artifacts were more common in the synthetic sequences (except proton-density), especially in synthetic STIR (Fig 3).

There were no statistically significant differences between readers regarding the scores assigned for image quality or lesion conspicuity. The Table summarizes these results.

\section{DISCUSSION}

The clinical use of synthetic MR imaging has focused mainly on brain pathology. The first mention of synthetic images possibly having a diagnostic value comparable with that of conventional sequences was in a study by Blystad et $\mathrm{al}^{4}$ in 2012. Other studies 


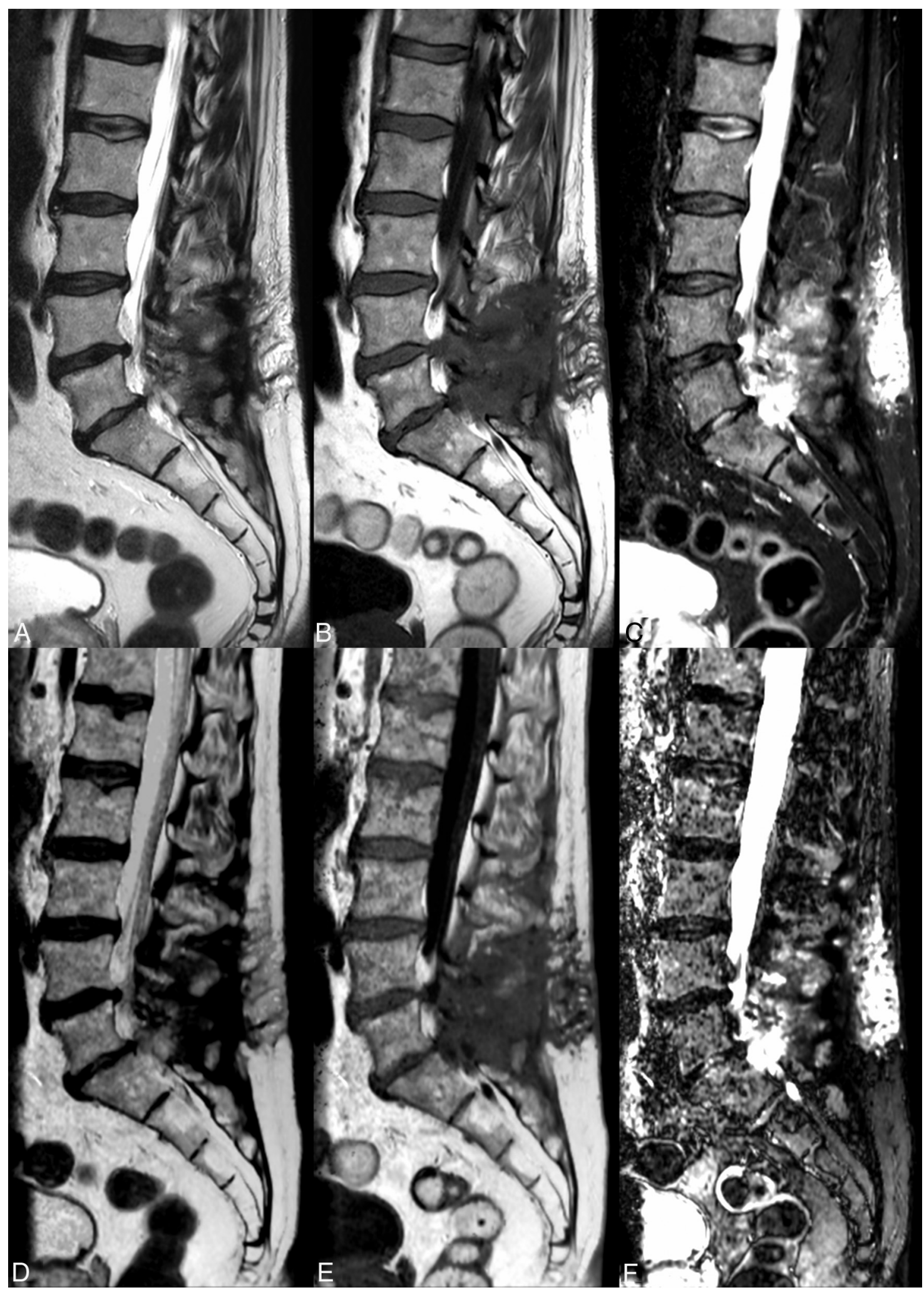

FIG 3. Conventional FSE T2 (A), spin-echo TT (B), and STIR $(C)$ images show a postsurgical lumbar spine with postoperative changes in the posterior soft tissues. The synthetic images $(D-F)$ depict these changes with similar detail. Note the dirty appearance of the vertebral bodies observed in the synthetic reconstructions and flow artifacts from the aorta, especially in the synthetic STIR sequence.

have since assessed the clinical feasibility of synthetic MR imaging across different neurologic conditions ${ }^{8,9}$ such as multiple sclerosis $^{10}$ and metastatic disease of the brain ${ }^{11}$ and in the pediatric population.
This technique has also been recently used for musculoskeletal imaging. A recent study, focusing on imaging of the normal knee, ${ }^{14}$ assessed the feasibility and diagnostic accuracy of synthetic MR imaging compared with conventional MR imaging as 
Median scores for image quality and the certainty of the presence or absence of a lesion and statistical differences between methods and readers ${ }^{a}$

\begin{tabular}{|c|c|c|c|c|c|c|c|c|}
\hline & \multicolumn{3}{|c|}{ Reader 1} & \multicolumn{3}{|c|}{ Reader 2} & \multicolumn{2}{|c|}{ Between Readers } \\
\hline & Conventional & Synthetic & $P$ & Conventional & Synthetic & $P$ & Conventional & Synthetic \\
\hline Image quality & 3 & 3 & .053 & 3 & 3 & .02 & 1.00 & .78 \\
\hline $\begin{array}{l}\text { Certainty of the presence or } \\
\text { absence of a lesion }\end{array}$ & 1 & 1 & .149 & 1 & 1 & .04 & 1.00 & .35 \\
\hline
\end{tabular}

a Scores for image quality: 1 = poor, 2 = fair, 3 = good; scores for the certainty of the presence or absence of a lesion: $0=$ not certain, $1=$ certain.

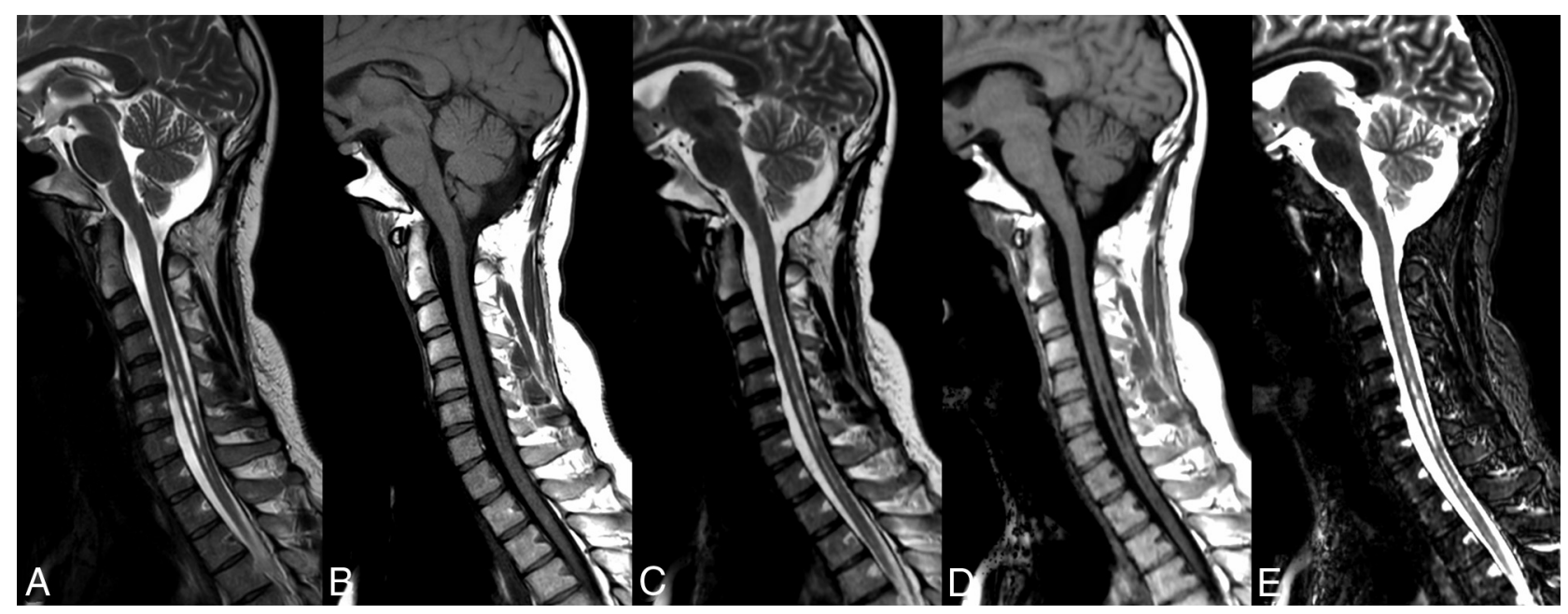

FIG 4. Sagittal conventional FSE T2 (A) and spin-echo $T 1(B)$ images and synthetic $T 2(C), T 1(D)$, and $S T I R(E)$ images illustrate a syrinx of the cervical cord. Note the same level of detail in both types of sequences.

well as the utility of synthetic quantitative T2 maps of the different knee structures compared with conventional T2 mapping sequences. The conclusion was that synthetic MR imaging provided comparable image quality and offered the potential to reduce the overall examination time. An exception was noted for bone marrow where the relative signal intensity and contrast of synthetic T1 images were lower than on conventional T1. Other studies focused on the feasibility of synthetic MR imaging for knee pathology. ${ }^{15,16}$ To our knowledge, this is the first study exploring the potential utility of synthetic MR imaging for spine and spinal cord imaging in clinical routine (Figs 4 and 5). In this pilot study, synthetic MR has been shown to be a feasible alternative or complement to conventional T1WI, T2WI, and STIR sequences in spine imaging. Image quality was considered not significant different from that produced by conventional sequences, and both diagnostic confidence and lesion conspicuity were at acceptable levels. These results are in agreement with previous reports stating that synthetic and conventional imaging have similar diagnostic utility. $^{4,6,9,10}$

Some differences were nevertheless observed between the 2 methods. The synthetic sequence, on occasion, had less contrast, a "dirty" appearance to the images, and less resolution (Fig 2), resulting in lower image-quality scores assigned by the readers in some cases. These differences were, however, not statistically significant and did not affect the overall perception of imaging quality or the diagnostic confidence. As previously reported, arbitrary signal changes found in the voxels containing 2 tissues that are particularly different are thought to be partial volume artifacts because they cannot be described using a monoexponential function. Consequently, fitted $\mathrm{T} 1$ and $\mathrm{T} 2$ relaxation appears as a combination of the 2 tissue values ${ }^{3,17}$ and can represent a limitation in synthetic imaging. In contrast, some authors described synthetic T1-weighted images having higher but not significantly different mean overall image-quality scores than conventional images and less subjective noise. ${ }^{6}$ This finding was due to the relatively low signal-to-noise ratio on conventional T1-weighted images.

The different backgrounds of the 2 readers (neuroradiology and musculoskeletal radiology, respectively) are probably the main factors affecting the lack of agreement observed in some cases. For example, some benign spine lesions, such as hemangiomas, were identified as abnormalities by reader 2 , but minimized by reader 1 .

With respect to the artifacts produced by synthetic MR imaging, it is known that synthetic FLAIR images of the brain have more pronounced artifacts, ${ }^{18}$ requiring adding conventional FLAIR sequences to the imaging protocol. At the level of the spine, image artifacts were particularly noticeable in the synthetic STIR sequence, pulsatile (vascular) flow artifacts (Fig 2) being the most frequent ones. Additionally, a dirty appearance to the images was observed in all synthetic sequences. Artifacts secondary to the presence of metal in the spine were identified on the synthetic sequences but were not more pronounced than in the conventional sequences (Fig 6).

These artifacts occurred more often in the dorsal and lumbar spine, where the synthetic images showed more noise compared with scans of the cervical spine, almost certainly due to a higher number of channels and coils used to image this area (spine and cervical coils versus spine coil only in the dorsolumbar region).

AJNR Am J Neuroradiol 39:1756-63 Sep 2018 www.ajnr.org 


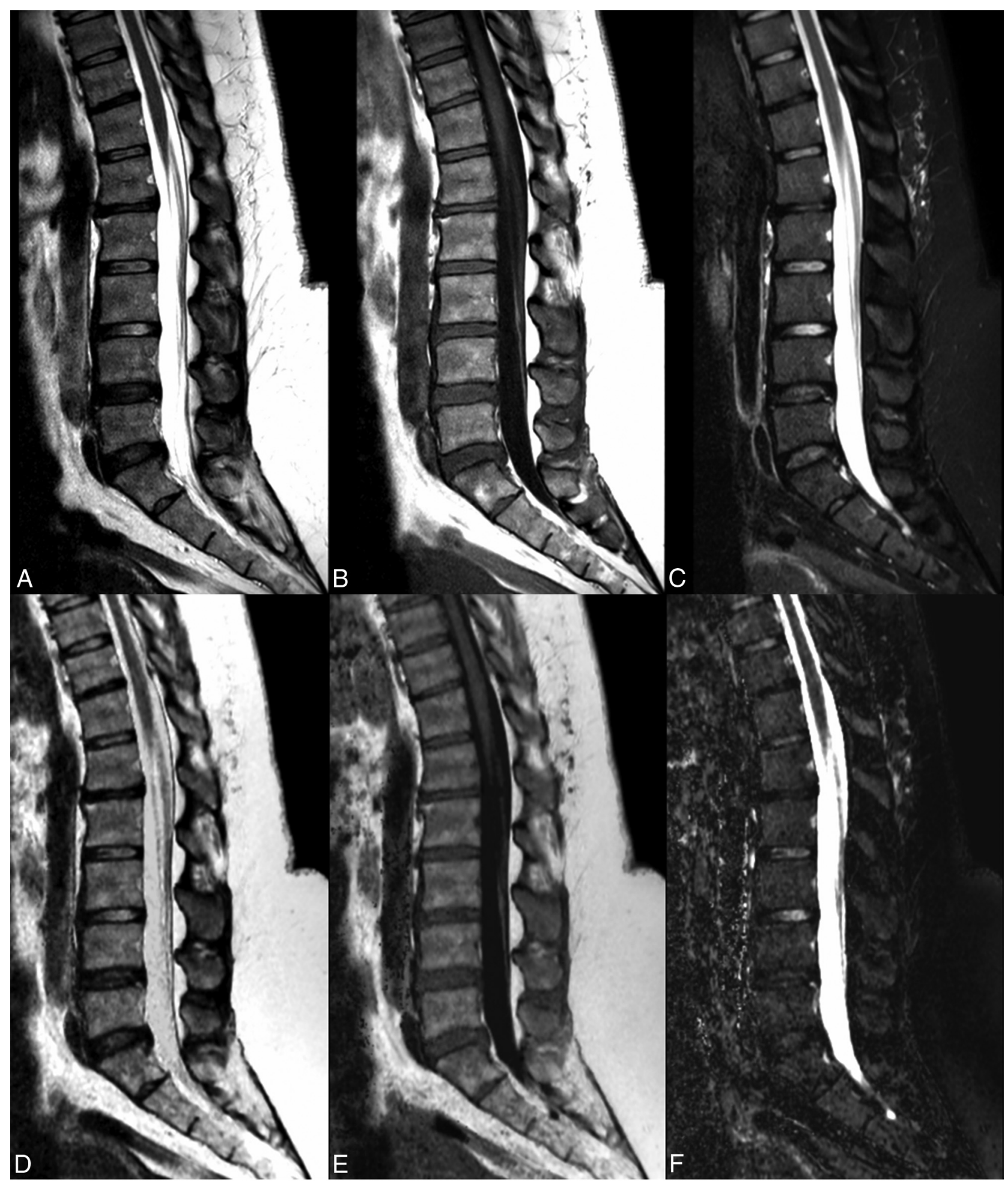

FIG 5. The normal dorsolumbar spine with conventional $(A-C)$ and synthetic $(D-F)$ sequences.

MR imaging time should be considered, especially in spine imaging due to the high prevalence of spinal pathology and, consequently, the high number of requests for MR imaging of this region. Furthermore, patients requiring spinal imaging frequently have back pain and find it more difficult to remain supine for long periods. Currently used imaging protocols for the spine have an average duration of approximately $30 \mathrm{~min}$ utes when the clinical indication does not warrant the use of contrast agent or only part of the spine needs to be imaged, as in disc herniation. When contrast administration is indicated or the whole spine needs to be imaged, the acquisition time is approximately 1 hour.

The SyntAc sequence was adapted for spine imaging and can produce at least 3 conventional sequences (T1, T2, STIR), which usually require an acquisition time of 11 minutes 20 seconds, offering the possibility of generating other contrasts if required. In 


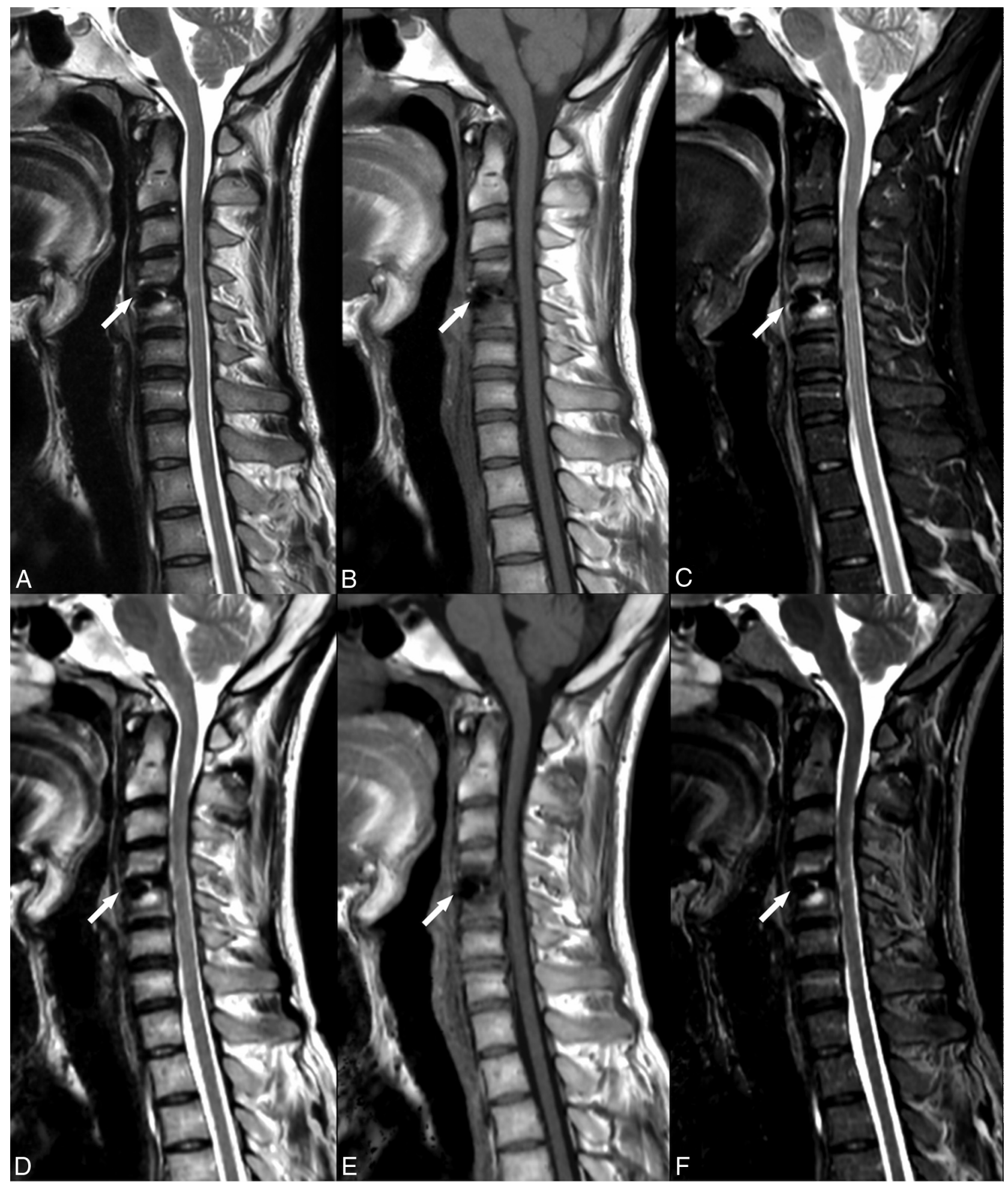

FIG 6. Sagittal conventional FSE T2 $(A)$, spin-echo T1 (B), and STIR $(C)$ sequences and corresponding synthetic sequences $(D-F)$ in a patient with an intervertebral cage (arrows). Note that the degree of magnetic susceptibility artifacts is the same in both sequences.

the present study, the acquisition time of synthetic MR imaging ( 5 minutes 40 seconds) was approximately half of that required for conventional sequences. Sagittal synthetic spine MR imaging could therefore potentially replace conventional sagittal T1, T2, and STIR sequences for imaging 1 spinal region (cervical, dorsal, or lumbar) and halve the acquisition time. However, if fat-satu- rated postcontrast sequences were needed, they would be obtained separately by conventional methods.

In addition to allowing shorter acquisition times, synthetic sequences provide quantitative $\mathrm{T} 1, \mathrm{~T} 2$, and proton-density maps (Fig 7). These can be used to derive relaxometry parameters of spinal components, ${ }^{19}$ thus providing quantitative information of AJNR Am J Neuroradiol 39:1756-63 Sep 2018 www.ajnr.org 


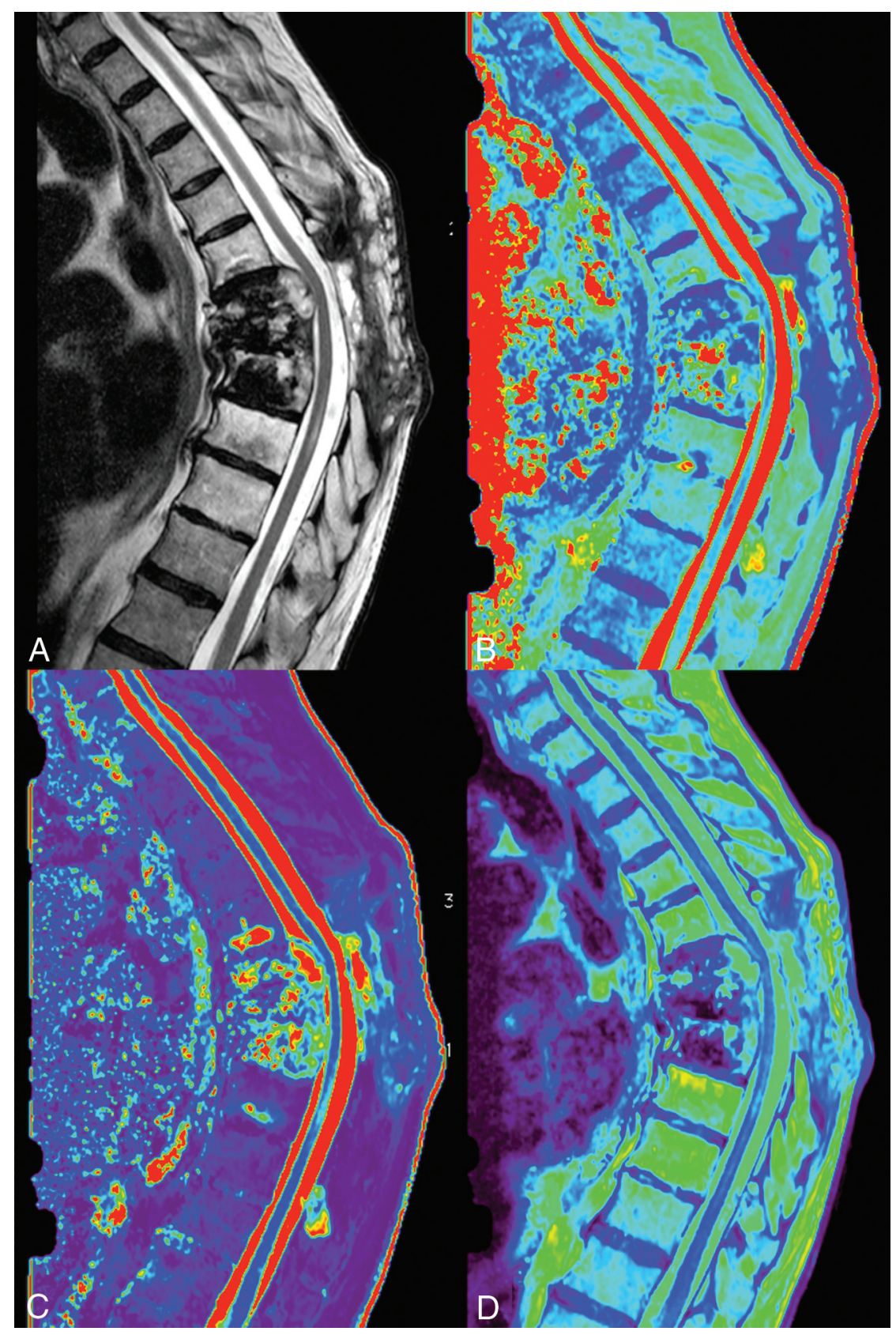

FIG 7. The dorsal spine in a patient with bone metastases illustrated by conventional FSE T2 (A) as well as T2 (B), T1 (C), and proton-density (D) maps.

interest for research and potentially clinical purposes with a view to using these quantified parameters daily in the assessment of common spine diseases (demyelinating diseases, degenerative conditions, spondylodiscitis).

In this study, identical TR and TE parameters were used in all patients to produce the synthetic sequences.

The main limitations of this study were the relatively small sample size and the limited number of pathologies in the patients included. From our observation, a compromise is still needed to increase the SNR while maintaining a reasonable acquisition time in the dorsolumbar region. This could potentially be improved with the implementation of this sequence on $3 \mathrm{~T}$ MR imaging. Conversely, in our study, the image quality and diagnostic confi- dence were very good in the cervical area, where the SyntAc sequence currently produces the best results.

We believe that our findings could facilitate the integration of quantitative MR imaging in clinical routine.

\section{CONCLUSIONS}

Synthetic MR imaging is feasible in spine imaging. However, some work and development are still required to improve synthetic STIR to reduce flow artifacts and increase the signal-tonoise ratio, particularly in the lumbar region. We believe that in the future, a significant reduction in acquisition time will be possible with this technique without sacrificing diagnostic accuracy. Furthermore, the quantitative information generated 
with this method will allow a novel approach to the diagnosis of spine disease.

\section{REFERENCES}

1. Liao C, Bilgic B, Manhard MK, et al. 3D MR fingerprinting with accelerated stack-of-spirals and hybrid sliding-window and GRAPPA reconstruction. Neuroimage 2017;162:13-22 CrossRef Medline

2. European Society of Radiology (ESR). Magnetic resonance fingerprinting: a promising new approach to obtain standardized imaging biomarkers from MRI. Insights Imaging 2015;6: 163-65 CrossRef Medline

3. Hagiwara A, Warntjes M, Hori M, et al. SyMRI of the brain: rapid quantification of relaxation rates and proton density, with synthetic MRI, automatic brain segmentation, and myelin measurement. Invest Radiol 2017;52:647-57 CrossRef Medline

4. Blystad I, Warntjes JB, Smedby O, et al. Synthetic MRI of the brain in a clinical setting. Acta Radiol 2012;53:1158-63 CrossRef Medline

5. Lee SM, Choi YH, You SK, et al. Age-related changes in tissue value properties in children: simultaneous quantification of relaxation times and proton density using synthetic magnetic resonance imaging. Invest Radiol 2018;53:236-45 CrossRef Medline

6. Lee SM, Choi YH, Cheon JE, et al. Image quality at synthetic brain magnetic resonance imaging in children. Pediatr Radiol 2017;47: 1638-47 CrossRef Medline

7. McAllister A, Leach J, West H, et al. Quantitative synthetic MRI in children: normative intracranial tissue segmentation values during development. AJNR Am J Neuroradiol 2017;38:2364-72 CrossRef Medline

8. Warntjes JB, Leinhard OD, West J, et al. Rapid magnetic resonance quantification on the brain: optimization for clinical usage. Magn Reson Med 2008;60:320-29 CrossRef Medline

9. Tanenbaum LN, Tsiouris AJ, Johnson AN, et al. Synthetic MRI for clinical neuroimaging: results of the Magnetic Resonance Image
Compilation (MAGiC) prospective, multicenter, multireader trial. AJNR Am J Neuroradiol 2017;38:1103-10 CrossRef Medline

10. Granberg T, Uppman M, Hashim F, et al. Clinical feasibility of synthetic MRI in multiple sclerosis: a diagnostic and volumetric validation study. AJNR Am J Neuroradiol 2016;37:1023-29 CrossRef Medline

11. Hagiwara A, Andica C, Hori M, et al. Synthetic MRI showed increased myelin partial volume in the white matter of a patient with Sturge-Weber syndrome. Neuroradiology 2017;59:1065-66 CrossRef Medline

12. Betts AM, Leach JL, Jones BV, et al. Brain imaging with synthetic MR in children: clinical quality assessment. Neuroradiology 2016;58: 1017-26 CrossRef Medline

13. West $\mathrm{H}$, Leach JL, Jones BV, et al. Clinical validation of synthetic brain MRI in children: initial experience. Neuroradiology 2017;59: 43-50 CrossRef Medline

14. Park S, Kwack KS, Lee YJ, et al. Initial experience with synthetic MRI of the knee at 3T: comparison with conventional T1 weighted imaging and T2 mapping. Br J Radiol 2017;90:20170350 CrossRef Medline

15. Yi J, Lee YH, Song HT, et al. Clinical feasibility of synthetic magnetic resonance imaging in the diagnosis of internal derangements of the knee. Korean J Radiol 2018;19:311-19 CrossRef Medline

16. Boudabbous S, Neroladaki A, Bagetakos I, et al. Feasibility of synthetic MRI in knee imaging in routine practice. Acta Radiol Open 2018;7:2058460118769686 CrossRef Medline

17. Whittall KP, MacKay AL, Li DK. Are mono-exponential fits to a few echoes sufficient to determine T2 relaxation for in vivo human brain? Magn Reson Med 1999;41:1255-57 CrossRef Medline

18. Vargas MI, Boto J, Delatre BM. Synthetic MR imaging sequence in daily clinical practice. AJNR Am J Neuroradiol 2016 Jul 21. [Epub ahead of print] CrossRef Medline

19. Drake-Pérez M, Delattre BM, Boto J, et al. Normal values of magnetic relaxation parameters of spine components with the synthetic MRI sequence. AJNR Am J Neuroradiol 2018;39:788-95 CrossRef Medline 